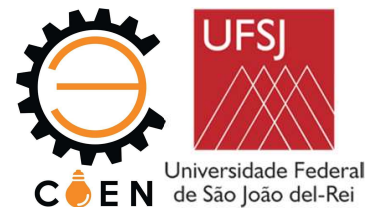

\title{
INFLUÊNCIA DE REGULADORES DE TENSÃO NO CÁLCULO DE CORRENTES DE CURTO CIRCUITO EM SISTEMAS DE DISTRIBUIÇÃO DE ENERGIA ELÉTRICA
}

\author{
Luís Henrique Rodrigues Diniz ${ }^{(1)}$ (luishenrique@eletroterraengenharia.com.br), Renan Souza Moura ${ }^{(2)}$ \\ (renan.moura@ifmg.edu.br), Ulysses Rondina Duarte ${ }^{(3)}$ (ulysses.rondina@ifmg.edu.br), \\ Carlos Renato Borges dos Santos ${ }^{(4)}$ (carlos.renato@ifmg.edu.br)
}

(1) (2) (3) (4) IFMG Campus Formiga - Departamento de Engenharia Elétrica - Rua Padre Alberico, 440, São Luiz, Formiga - MG.

RESUMO: Este artigo aborda a presença de correntes de curto circuito variáveis em sistemas automatizados de distribuição de energia elétrica. Usualmente, os ajustes do sistema de proteção são fixos, logo, podem não contemplar qualquer variação de corrente durante a coordenação. Entretanto, a inserção de um regulador de tensão altera a impedância do sistema em função da posição do tap e isso pode comprometer a atuação correta de relés que visam à proteção do sistema elétrico. Diante disto, neste trabalho é analisada a influência das correntes de curto circuito variáveis em função do carregamento do sistema e propõe três métodos computacionais, simulados em ambiente Matlab ${ }^{\circledR}$, objetivando o controle e atenuação destas correntes: i) o redespacho de potência reativa; ii) a inserção de reatores em série e; iii) a comutação eletrônica de taps. Em todas as simulações, os limites operacionais do sistema analisado são considerados. Os resultados demonstraram que todos os métodos são eficazes para reduzir as correntes de curto circuito variáveis: método i) em torno de $17 \%$, método ii) em torno de $5 \%$ e método iii) em torno de $19 \%$. Vantagens e desvantagens de cada método são discutidas neste artigo. Embora os métodos computacionais apresentados ainda não sejam passiveis de serem empregados em sistemas experimentais de larga escala, espera-se que este trabalho fomente uma maior atenção ao tema de ajuste dinâmico do sistema de proteção, principalmente com a intensificação dos investimentos em smart grids.

PALAVRAS-CHAVE: Correntes de curto circuito variáveis. Curva PV. Métodos de controle de correntes de curto circuito.

\section{INTRODUÇÃO}

O avanço tecnológico da humanidade resulta em aumentos contínuos na demanda de energia elétrica. Logo, caracteriza-se como principal função dos sistemas elétricos de potência (SEP) suprir esta necessidade crescente de energia elétrica de forma segura, confiável e economicamente viável (FURINI, 2008).

Contudo, estes sistemas estão sujeitos a diversos distúrbios que podem ocasionar situações de instabilidade como: variações na carga atendida, inserção ou remoção de equipamentos shunt, curtos circuitos, dentre outros.

Em razão disto, é grande a preocupação em garantir que as condições operativas das cargas sejam atendidas. Assim, para manter os níveis especificados de tensão para o consumidor e como forma de evitar instabilidade na rede de distribuição, grande parte dos sistemas atuais utilizam reguladores de tensão e um sistema de proteção apropriado.

O sistema de proteção convencional leva em conta apenas um valor crítico de atendimento de cargas residenciais, comerciais e industriais para dimensionamento dos dispositivos, além de considerar apenas o fluxo de potência em operação radial, no sentido da fonte para as cargas (GERS \& HOLMES, 2005). O uso de reguladores se deve ao fato de possuir, relativamente, um baixo custo de implementação e flexibilidade operacional, fatores que proporcionam a realização de ajustes

IX COEN - Congresso de Engenharias da UFSJ Interconexão. 

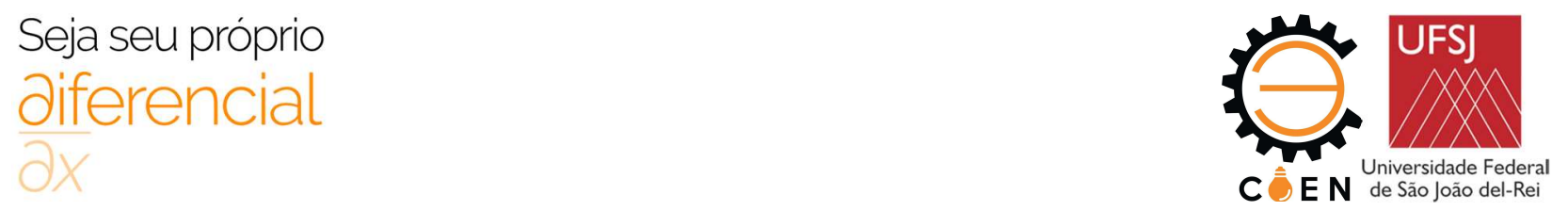

automáticos sob diversas condições de carregamento da rede (MEDEIROS \& PIMENTEL, 2002). Agem com a atuação de taps em função da tensão de referência.

Entretanto a característica operativa de um regulador de tensão de manter o nível de tensão com a mudança de taps também resulta na modificação da impedância da rede elétrica. Mais precisamente, para cada posição de tap do regulador de tensão, existirá uma matriz de impedância/admitância da rede associada. Tal fato tende a deixar a abordagem do sistema de proteção convencional obsoleta devido à dinâmica do carregamento ao longo de um período, aumento de cargas não lineares e o advento da geração distribuída.

De Almeida (2012) enfatiza que a operação dinâmica do sistema faz com que surjam correntes de curto circuito variáveis, que podem não ter sido contempladas no projeto do sistema de proteção, podendo causar sérios danos à estrutura, ao ambiente e aos seres vivos, dentre os quais os humanos.

Dentro deste contexto, este trabalho ressalta o surgimento de correntes de curto circuito variáveis em sistemas automatizados de distribuição de energia elétrica. Após a detecção destas correntes em um sistema com regulador de tensão, é proposto três formas de atenuação destas correntes por meio de simulações em MATLAB. Embora tais propostas de atenuação de correntes de curto circuito ainda não sejam aplicáveis dentro da rotina operacional de um sistema de distribuição de energia elétrica, espera-se que este artigo incentive futuros estudos sobre o tema, pois a inserção de equipamentos automatizados produzirá novos desafios técnicos como os demonstrados nas próximas seções.

A estrutura deste trabalho é a seguinte: a seção 1 é responsável por realizar uma apresentação geral do tema para que os objetivos sejam anunciados na seção 2 . A seção 3 aborda os principais conceitos para um melhor entendimento da metodologia proposta na seção 4 . Por fim, os resultados obtidos e as considerações finais estão contidas nas seções 5 e 6 , respectivamente.

\section{OBJETIVOS}

Os objetivos gerais deste artigo são demonstrar a presença de correntes de curto circuito variáveis em sistemas de distribuição automatizados e propor, por meio de simulações computacionais, três métodos dinâmicos de controle e atenuação para tais correntes.

\section{REFERENCIAL TEÓRICO}

Como forma de propor um melhor entendimento deste artigo, um breve referencial teórico será apresentado nas próximas seções, abordando as seguintes definições:

\subsection{Sistemas de Distribuição de Energia Elétrica}

O sistema tem início a partir de subestações locais, que realizam a redução dos níveis de tensão oriundos das linhas de transmissão. Salvo em casos específicos, a estrutura da rede é radial, isto é, o fluxo de potência é feito em apenas uma direção, das subestações direto para o consumidor final.

Um fator preponderante ao processo é o conhecimento da carga que será atendida, pois o carregamento influi em vários aspectos na linha de distribuição, como por exemplo, na determinação do nível de tensão de operação.

Ainda na análise das cargas, é possível afirmar que este carregamento é desbalanceado, pois um sistema de distribuição apresenta majoritariamente cargas monofásicas e bifásicas. Além disso, o

IX COEN - Congresso de Engenharias da UFSJ Interconexão. 


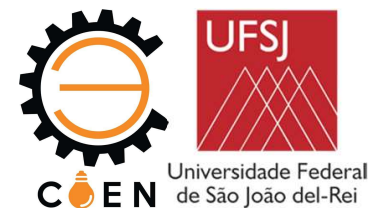

alimentador de um sistema de distribuição de energia elétrica atende uma demanda variável de energia ao longo do dia, conforme ilustrado pela Figura 1.

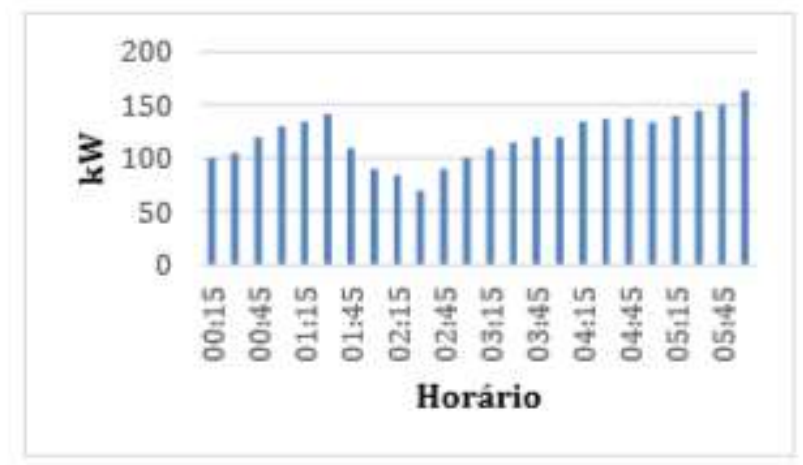

Figura 1. Curva de demanda diária. Fonte: Autores

Outro problema relacionado às cargas são as faltas de energia, uma vez que as redes de distribuição estão mais propensas a isto, devido à sua estrutura radial que possibilita um único sentido para o fluxo de potência.

Em função destes fatores, é preciso realizar uma modelagem do sistema de distribuição como forma de prever situações reais por meio de simulações, buscando uma otimização do processo, de forma que proporcione a entrega de tensão e potência aos consumidores de forma contínua, segura e com qualidade.

\subsection{Reguladores de tensão}

A estrutura básica de um regulador pode ser vista na Figura 2.

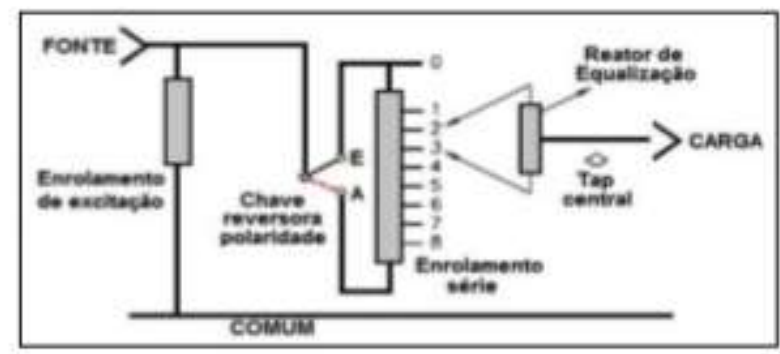

Figura 2. Estruturação de um regulador de tensão. Fonte: (BURATTI, 2016).

Em virtude da busca do fornecimento de energia elétrica atendendo os critérios de qualidade de energia impostos pela ANEEL por meio do PRODIST Módulo 8, concessionárias têm optado pela inserção de reguladores de tensão na rede, como forma de assegurar a tensão no barramento de carga em níveis adequados.

O regulador de tensão é um equipamento que, ao ser instalado na rede, mantém um nível de tensão pré-determinado no ponto de regulação, apesar das variações das cargas, desde que sua margem de regulação não seja ultrapassada. Construtivamente, o regulador é composto de um autotransformador, um comutador automático de derivação (tap) sob carga e de um sistema de controle, que corrige os desvios momentâneos da tensão de regulação, relativos ao valor especificado. Usualmente, os reguladores para aplicação em linhas de distribuição são equipamentos monofásicos.

IX COEN - Congresso de Engenharias da UFSJ Interconexão. 


\section{Seja seu próprio

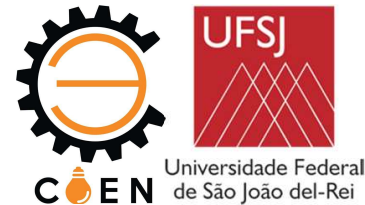

Sua instalação é realizada através da formação de bancos trifásicos (MEDEIROS \& PIMENTEL, 2002).

Uma vez que o comportamento destes equipamentos está sujeito a variações contínuas de forma a manter a estabilidade do sistema, independentemente do valor de potência requerido pela carga, percebe-se que é incorporada uma dinâmica no processo (MOURA et al., 2016).

Além do mais, à cada acréscimo de tap realizado, a impedância equivalente do sistema aumenta substancialmente em relação à um sistema sem a presença de reguladores, já que a impedância da linha de distribuição é baixa.

\subsection{Curva PV}

Esta ferramenta obtém, por meio de sucessivos fluxos de potência, uma curva do comportamento da tensão em função do crescimento de carga, isto é, a relação da magnitude de tensão em um barramento com o nível de carregamento atendido por este mesmo barramento (CONDEGA, 2013).

Ao realizar incrementos contínuos de carga, permite-se analisar diferentes condições operativas do sistema e possibilita a obtenção do ponto de máxima transferência de potência, também chamado de valor de máximo carregamento (KUNDUR, 2004).

A Figura 3 demonstra uma curva genérica com seus principais pontos em destaque, sendo que o eixo $\mathrm{Y}$ corresponde à tensão e o eixo $\mathrm{X}$ corresponde à potência na carga. Conforme ilustrado pela Figura 3, o $P_{C}$ corresponde ao ponto crítico, aquele o qual o sistema entrega a máxima transferência de potência em nível de tensão crítica. A distância entre o ponto operativo nominal e o ponto crítico é definido como margem de carga (DE SOUZA et al., 2011).

Além disso, para qualquer valor de carga atendida existem dois pontos operativos: um estável e um instável. O ponto A indicado na Figura 3 é estável, pois qualquer incremento de carga é acompanhado de um declínio no nível de tensão. O ponto B, também representado na Figura 3, é do tipo instável, pois o aumento de carga resulta em elevação da tensão de operação. Além disso, sugerese sempre evitar trabalhar em torno do ponto B, pois uma tensão em níveis críticos, segundo o PRODIST 8 (2018), resulta em uma corrente elevada, maiores quedas de tensão e baixo fator de potência.

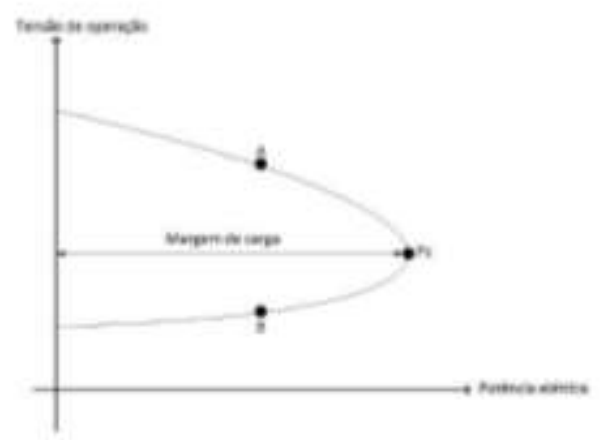

Figura 3. Curva PV genérica. Fonte: Autores

IX COEN - Congresso de Engenharias da UFSJ Interconexão. 


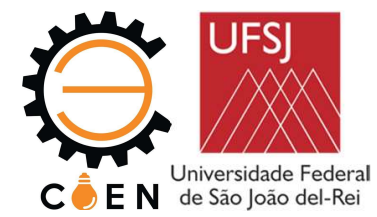

\subsection{Cálculos de correntes de curto circuito}

A definição de curto circuito - ou falta elétrica - está relacionada a uma conexão de natureza intencional ou acidental, comumente de baixa impedância, entre dois ou mais pontos que, geralmente, estão em diferentes potenciais elétricos, resultando em uma corrente elétrica de magnitude elevada e tensão quase nula no ponto de falta, variando a amplitude de acordo com o tipo de curto circuito (OSORIO \& PAN, 2018).

Para modelar matematicamente os curtos circuitos em sistemas de distribuição é necessário deduzir expressões próprias para este sistema utilizando a estrutura de fase. Assim, o cálculo da corrente consiste basicamente em obter-se o circuito equivalente de Thèvenin, com a impedância acumulada desde a geração até o ponto de defeito (KERSTING, 2002). A Figura 4 exemplifica o circuito equivalente com um nó em curto.

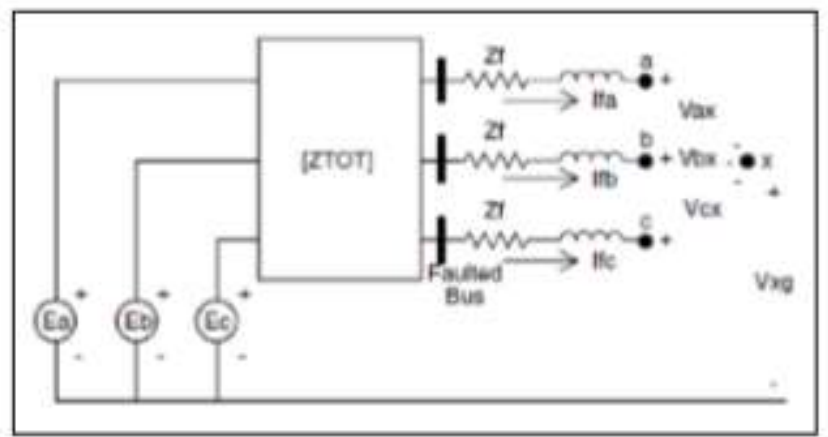

Figura 4. Circuito equivalente de Thèvenin. Fonte: (KERSTING, 2002).

$\mathrm{Na}$ Figura 4 as tensões $E a, E b$ e $E c$ equivalem às tensões fase-terra equivalentes de Thèvenin no ponto com falta, a matriz [ZTOT] representa a matriz de impedância de fase equivalente de Thèvenin no ponto com falta, de tal modo que $Z f$ corresponde à impedância de falta.

\section{METODOLOGIA}

Partindo dos conceitos supracitados, é apresentada a metodologia implementada para a realização das simulações propostas. Todas as abordagens são realizadas por meio do software Matlab ${ }^{\circledR}$ e são fundamentadas seguindo literaturas conceituadas.

A fim de demonstrar a influência da automação no processo de distribuição de energia, a primeira etapa trata-se de uma comparação de um sistema sem a presença de dispositivos automatizados com este mesmo sistema após a inserção de um regulador de tensão. Foi escolhido como sistema base um sistema teste do IEEE para simulações computacionais. Este sistema foi adaptado para atender às alterações da automação da rede.

Esta adaptação do sistema consiste na inserção de um dispositivo automatizado responsável pela regulação dos níveis de tensão no barramento de carga. O regulador foi inserido após o secundário do transformador, logo, foi necessário criar uma barra simbólica entre os dois equipamentos, denominada 3r. O circuito adaptado é mostrado na Figura 5. 


\section{Seja seu próprio Diferencial}
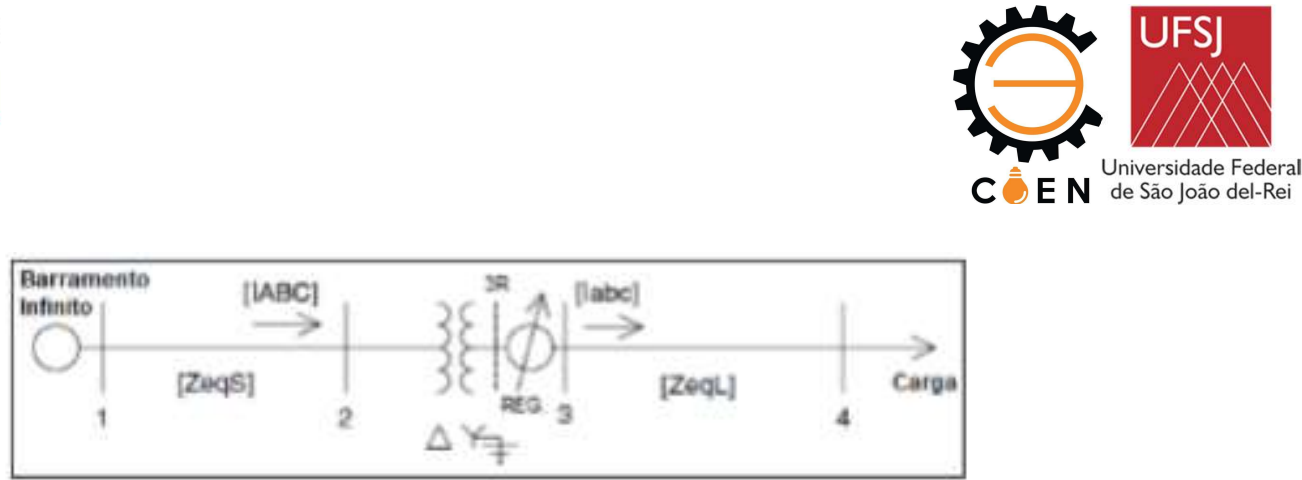

Figura 5. Sistema "IEEE 4 Node Test Feeder" adaptado.

Em seguida é plotada a curva PV tanto para o sistema sem a presença de dispositivos automatizados, quanto para o sistema com saída de tensão controlada por regulador. A comparação entre os sistemas também é realizada nos cálculos de correntes de curto circuito. $O$ intuito é verificar a alteração do comportamento da corrente à medida que o carregamento aumenta.

Ainda no processo de cálculo das correntes, são aplicados diferentes tipos de faltas elétricas a fim de verificar se a alteração do tipo de curto circuito ocorrido também gera variação na magnitude das correntes de curto circuito.

Com estes dados em mãos, aplica-se a metodologia desenvolvida para atenuação das correntes de curto circuito em sistemas automatizados. Três métodos são apresentados de forma que possibilite a comparação de seus respectivos resultados.

O primeiro método consiste no redespacho de reativos para a rede de distribuição no momento do curto. Para este método aplicou-se uma corrente capacitiva contrária ao fluxo de corrente de fuga.

O segundo método é realizado por meio de reatores em série com a linha de distribuição, os quais representam uma reatância indutiva, responsável pela oposição oferecida ao fluxo de corrente alternada que circula pela rede.

Por fim, o terceiro método utiliza-se do princípio de funcionamento do regulador de tensão, mais especificamente do chaveamento do comutador. No instante da falta, o tap atual é deslocado instantaneamente para o maior valor de tap, aumentando a impedância própria da rede no instante da falta. Lembrando que só é possível este deslocamento instantâneo devido ao comutador eletrônico, onde tiristores substituem as chaves eletromecânicas usuais, proporcionando a mudança de taps sem a necessidade de excursionar por cada nível intermediário das chaves.

\section{RESULTADOS E DISCUSSÕES}

Com base na metodologia apresentada, esta seção visa a apresentação dos resultados obtidos, bem como a análise sobre os valores relevantes para a compreensão da proposta estabelecida para este artigo.

\subsection{Curva PV}

Para realizar os fluxos de potência trifásicos necessários para confecção das curvas foram consideradas todas as características construtivas dos sistemas testes do IEEE. É salientado que ambos sistemas seguiram os mesmos procedimentos de simulação.

Uma vez que se trata de um sistema de distribuição desbalanceado, cada fase atende à uma carga de potência distinta. Sendo assim, as Figuras 6,7 e 8 confrontam as fases A, B e C, sem a presença de regulador de tensão e após a inserção do mesmo, a fim de verificar a influência deste equipamento no comportamento do sistema. A margem de carga sem e com a presença do regulador de tensão valem, respectivamente, 3,1 [p.u.] e 2,4 [p.u.].

IX COEN - Congresso de Engenharias da UFSJ Interconexão. 
Seja seu próprio diferencial
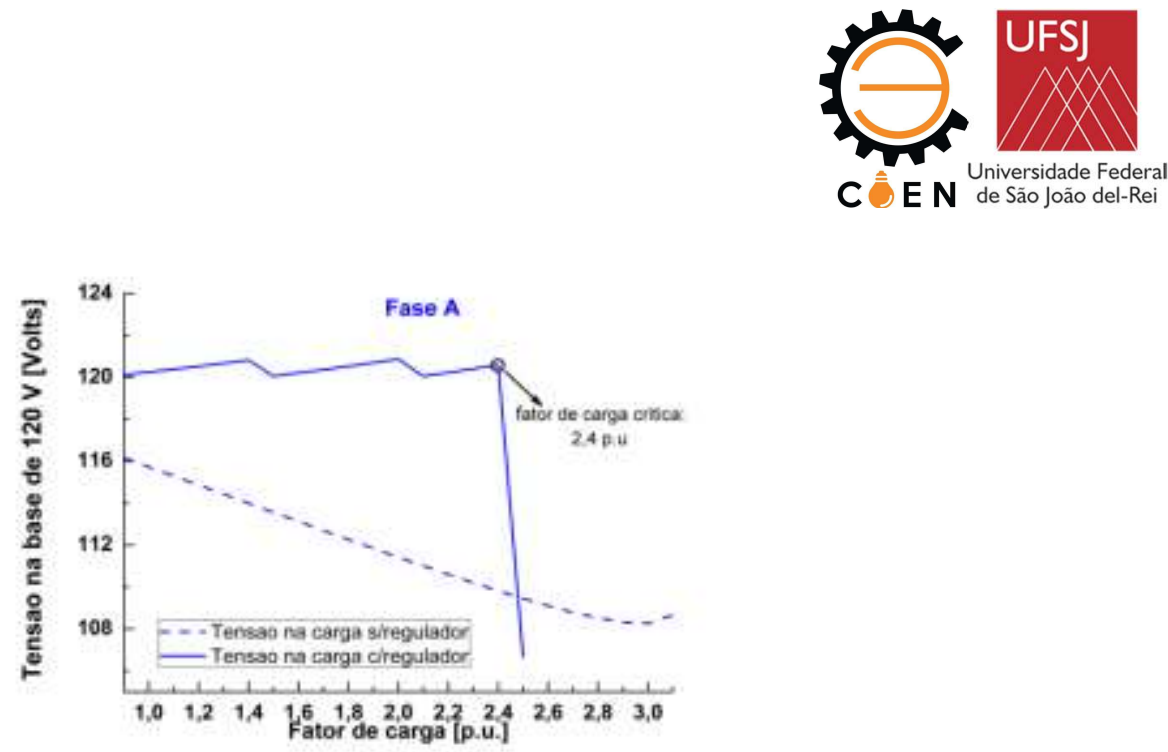

Figura 6. Curvas PV da fase A.

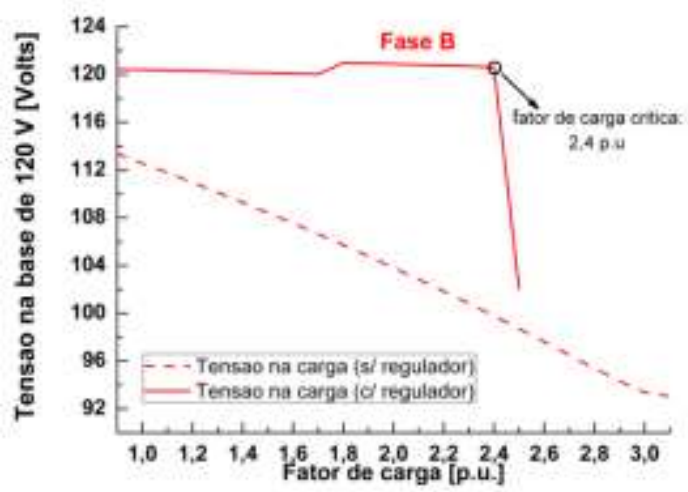

Figura 7. Curvas PV da fase B.

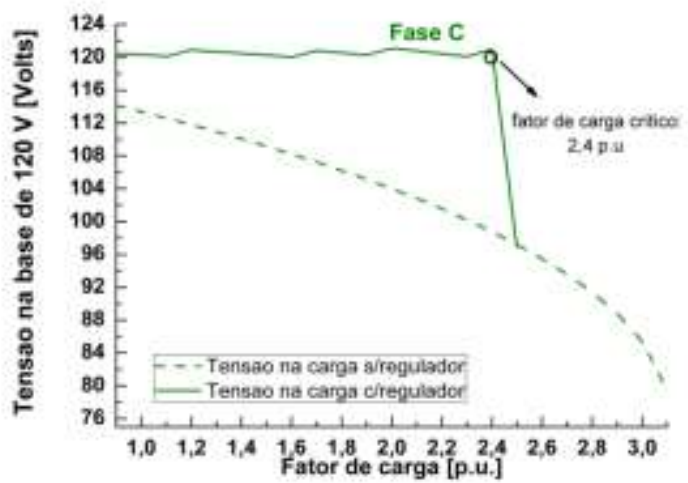

Figura 8. Curvas PV da fase C.

A partir dos resultados obtidos pelas Figuras 6 a 8, é possível esboçar as Tabelas 1 e 2 com os valores mais significativos das curvas, estabelecendo três fatores de carregamento (leve, moderado, máximo). 

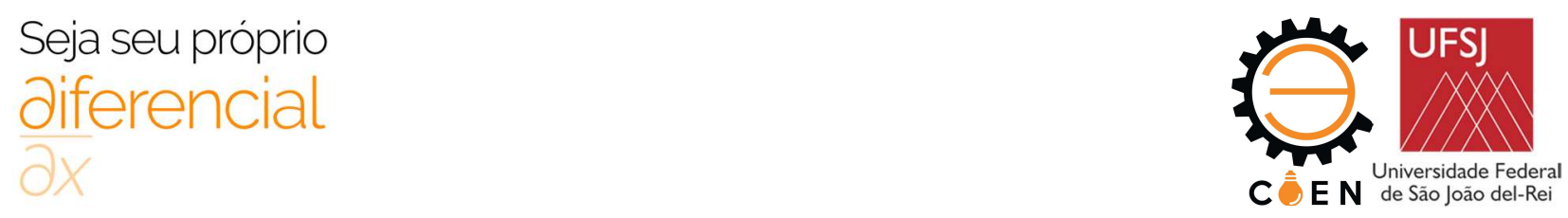

Tabela 1. Tensão na carga no sistema sem regulador de tensão.

\begin{tabular}{ccc|ccc|ccc}
\hline \multicolumn{6}{c}{ Tensão no barramento de carga em função do carregamento [Volts] } \\
\hline \multicolumn{2}{c|}{ Carga leve (0,9 p.u.) } & \multicolumn{3}{c|}{ Carga moderada (1,7 p.u.) } & \multicolumn{3}{c}{ Carga máxima $(3,1$ p.u.) } \\
\hline Fase A & Fase B & Fase C & Fase A & Fase B & Fase C & Fase A & Fase B & Fase C \\
\hline \multirow{2}{*}{116,2} & 113,4 & 114,1 & 112,7 & 106,6 & 107,2 & 108,7 & 93,05 & 79,44 \\
\hline
\end{tabular}

Tabela 2. Tensão na carga no sistema adaptado com regulador de tensão.

\begin{tabular}{|c|c|c|c|c|c|c|c|c|}
\hline \multicolumn{9}{|c|}{ Tensào no barramento de carga em função do carregamento [Volts] } \\
\hline \multicolumn{3}{|c|}{ Carga leve ( 0,9 p.u. $)$} & \multicolumn{3}{|c|}{ Carga moderada (1,7 p.u.) } & \multicolumn{3}{|c|}{ Carga máxima ( 2,4 p.u.) } \\
\hline Fase A & Fase B & Fase $\mathrm{C}$ & Fase $\mathrm{A}$ & Fase B & Fase C & Fase A & Fase B & Fase C \\
\hline 120,13 & 120,44 & 120,48 & 120,37 & 120,02 & 120,82 & 120,61 & 120,62 & 120,96 \\
\hline
\end{tabular}

Pela disposição dos dados da Tabela 1, verifica-se que, na prática, o primeiro sistema, sem a presença de reguladores de tensão, é totalmente inviável do ponto de vista de estabilidade e qualidade de energia, e por consequência, econômico. O que justifica esta afirmação é definição de que níveis de tensão abaixo de $90 \%$ são consideradas críticas pelo PRODIST Módulo 8.

Em contrapartida, o segundo sistema, Tabela 2, consegue, com a presença do regulador de tensão, operar com estabilidade em todos os pontos de carregamento, visto que a tensão permanece na ordem de $120 \mathrm{~V}$ (secundário do transformador de corrente) nos três pontos de análise. Isto sugere que os sistemas de distribuição atuais devem ter em sua estrutura dispositivos autônomos capazes de fazer a leitura do estado de tensão na carga e realizar a correção de forma automática.

Como ponto negativo dos sistemas automatizados tem-se que ao passo que a potência do barramento de carga se eleva, a corrente para supri-la também aumenta. Como o regulador de passo de tensão faz a leitura da queda de tensão na linha entre o regulador e o centro de carga, o fato de circularem correntes de maiores amplitudes faz com que a queda seja cada vez maior, gerando maiores esforços do equipamento no circuito interno de queda de tensão. Desta forma, o limite máximo de operação será menor se comparado ao sistema 1 (sem o regulador), sendo este máximo carregamento de valor igual à 2,4 p.u.

\subsection{Correntes de curto circuito}

A análise de corrente de curto circuito é feita a fim de compreender a relação entre carregamento do sistema e magnitude da corrente de falta. Partindo do pressuposto que os sistemas de alta confiabilidade são controlados por reguladores, é verificado se a presença de reguladores afeta na amplitude da corrente. As Tabelas 3 e 4 demonstram as correntes de curto circuito obtidas após a simulação de uma falta elétrica entre as fases A e B, para o sistema sem regulador e após a inserção do mesmo, respectivamente. 

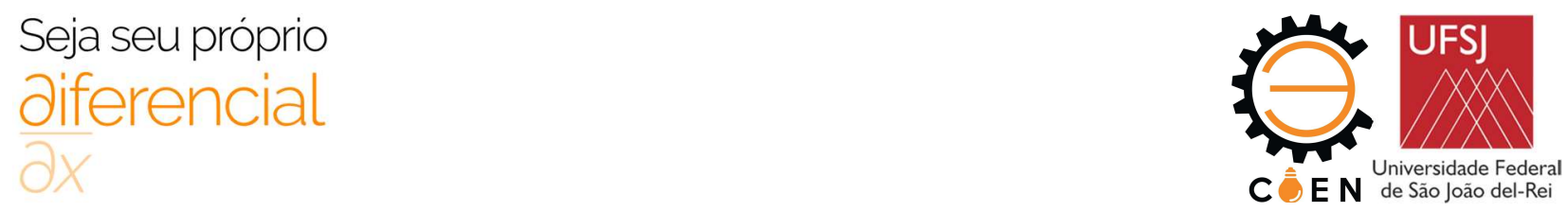

Tabela 3. Correntes de curto circuito para o sistema sem regulador de tensão.

\begin{tabular}{ccc|ccc|ccc}
\hline \multicolumn{7}{c}{ Correntes de curto circuito entre as fases A e B [A] } \\
\hline \multicolumn{2}{c|}{ Carga leve $(0,9$ p.u) } & \multicolumn{3}{c|}{ Carga moderada (1,7 p.u) } & \multicolumn{3}{c}{ Carga maxima $(2,4$ p.u $)$} \\
\hline Fase A & Fase B & Fase C & Fase A & Fase B & Fase C & Fase A & Fase B & Fase C \\
\hline & & & & & & & & \\
$4.194,7$ & $4.194,7$ & 0 & $4.194,7$ & $4.194,7$ & 0 & $4.194,7$ & $4.194,7$ & 0 \\
\hline
\end{tabular}

Tabela 4. Correntes de curto circuito para o sistema com regulador de tensão.

\begin{tabular}{|c|c|c|c|c|c|c|c|c|}
\hline \multicolumn{9}{|c|}{ Correntes de curto circuito entre as fases $A$ e $B$ [A] } \\
\hline \multicolumn{3}{|c|}{ Carga leve ( 0,9 p.u.) } & \multicolumn{3}{|c|}{ Carga moderada ( 1,7 p.u.) } & \multicolumn{3}{|c|}{ Carga máxima (2,4 p.u.) } \\
\hline Fase A & Fase B & Fase C & Fase A & Fase B & Fase C & Fase A & Fase B & Fase C \\
\hline 565,81 & 565,81 & 0 & 570,74 & 570,74 & 0 & 576,73 & 576,73 & 0 \\
\hline
\end{tabular}

Os dados expostos pela Tabela 3 mostram que sistemas onde não há presença de reguladores de tensão, o carregamento não exerce influência sobre a corrente de curto circuito. Isto deve-se ao fato de que, se a falta elétrica ocorre antes do barramento de carga, a corrente do sistema tende a percorrer o caminho de menor impedância, fazendo com que não exista fluxo de corrente para a carga. Deste modo, como a impedância de Thèvenin (ZTH) permanece a mesma para todos os pontos, as correntes de curto circuito serão de mesma amplitude (no valor de 4.194,7 [A]).

Por sua vez, ao analisar a Tabela 4 evidencia-se que o carregamento passa a influenciar, mesmo que indiretamente, na corrente de curto circuito, pois existe uma variação na amplitude que não era visualizada no sistema sem regulador.

Também se nota que houve uma diminuição da magnitude da corrente de curto circuito indicada pela Tabela 4 em comparação com a Tabela 3. Este fato ocorre devido ao módulo da impedância do regulador ser muito maior do que as impedâncias do sistema anterior. Logo, como todos os equipamentos estão dispostos em série, o regulador gera um acréscimo significativo à impedância equivalente de Thèvenin. A Tabela 5 indica o módulo da impedância do regulador em função da posição do tap para cada fase.

Tabela 5. Variação da impedância do regulador conforme a mudança de posição dos taps.

\begin{tabular}{c|c|c|c|c|c|c|c|c|c|c}
\hline \multicolumn{1}{c}{ Módulo da impedância do regulador de tensão [Q] } \\
\hline TAP 0 & TAP 1 & TAP 2 & TAP 3 & TAP 4 & TAP 5 & TAP 6 & TAP 7 & TAP 8 & TAP 9 & TAP 10 \\
\hline & 3,2005 & 3,2687 & 3,3369 & 3,4051 & 3,4733 & 3,5415 & 3,6097 & 3,6779 & 3,7461 & 3,8143 \\
\hline
\end{tabular}

Também são feitas as simulações aplicando tipos de faltas diferentes ao sistema automatizado, como forma de verificar se existe novamente variação de correntes de curto circuito em função do carregamento do sistema elétrico.

Sendo assim, são apresentadas as Tabelas 6,7 e 8, referentes às faltas fase-terra, fase-faseterra e fase-fase-fase, respectivamente.

IX COEN - Congresso de Engenharias da UFSJ Interconexão. 


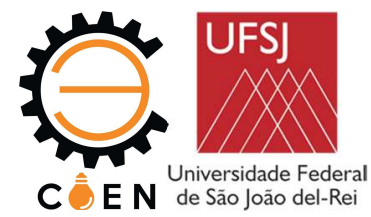

Tabela 6. Correntes de curto circuito para uma falta fase-terra.

\begin{tabular}{|c|c|c|c|c|c|c|c|c|}
\hline \multicolumn{9}{|c|}{ Correntes de curto circuito da fase $\mathrm{A}$ para a terra [A] } \\
\hline \multicolumn{3}{|c|}{ Carga leve ( 0,9 p.u.) } & \multicolumn{3}{|c|}{ Carga moderada ( 1,7 p.u. $)$} & \multicolumn{3}{|c|}{ Carga máxima ( 2,4 p.u. $)$} \\
\hline Fase A & Fase B & Fase C & Fase A & Fase B & Fase $\mathrm{C}$ & Fase A & Fase B & Fase C \\
\hline 613,82 & 0 & 0 & 620,34 & 0 & 0 & 625,64 & 0 & 0 \\
\hline
\end{tabular}

Para a falta do tipo fase-terra, evidencia-se que o curto com fuga para a terra aumenta consideravelmente a amplitude da corrente de curto circuito, em relação ao curto circuito apresentado na Tabela 4, fazendo com que a proteção tenha que atuar para valores bem acima dos obtidos quando consideradas apenas as faltas entre fases.

Tabela 7. Correntes de curto circuito para uma falta fase-fase-terra.

\begin{tabular}{ccc|ccc|ccc}
\hline \multicolumn{6}{c}{ Correntes de curto circuito entre as fases A e B para a terra [A] } \\
\hline \multicolumn{2}{c|}{ Carga leve (0,9 p.u.) } & \multicolumn{3}{c|}{ Carga moderada (1,7 p.u.) } & \multicolumn{3}{c}{ Carga máxima (2,4 p.u.) } \\
\hline Fase A & Fase B & Fase C & Fase A & Fase B & Fase C & Fase A & Fase B & Fase C \\
\hline & & & & & & & & \\
624,20 & 638,25 & 0 & 627,25 & 641,13 & 0 & 629,66 & 643,36 & 0 \\
\hline
\end{tabular}

Nota-se que para uma falta entre duas fases e a terra, as correntes de curto circuito entre as fases deixam de ser simétricas. Este fato é justificado pela inexistência de transposição em linhas de distribuição, o que resulta em desequilíbrios devido às impedâncias mútuas diferentes entre as fases. Além disso, é evidente que a assimétrica de correntes de curto circuito sugere que dispositivos com parametrização de proteção iguais para as três fases podem resultar em falhas e/ou danos ao sistema.

Tabela 8. Correntes de curto circuito para uma falta fase-fase-fase.

\begin{tabular}{ccc|ccc|ccc}
\hline \multicolumn{6}{c}{ Correntes de curto circuito entre as fases A, B e C [A] } \\
\hline \multicolumn{2}{c|}{ Carga leve (0,9 p.u.) } & \multicolumn{2}{c|}{ Carga moderada (1,7 p.u.) } & \multicolumn{3}{c}{ Carga máxima (2,4 p.u.) } \\
\hline Fase A & Fase B & Fase C & Fase A & Fase B & Fase C & Fase A & Fase B & Fase C \\
\hline & & & & & & & & \\
640,54 & 649,43 & 639,71 & 643,62 & 652,55 & 642,72 & 646,03 & 654,99 & 645,07 \\
& & & & & & & & \\
\hline
\end{tabular}

Verifica-se que o curto circuito trifásico, Tabela 8, apresenta as maiores amplitudes de corrente, fato este que demonstra que este tipo de falta elétrica, apesar de ser a mais rara, pode representar os maiores riscos ao sistema.

Os resultados contidos nas Tabelas 4, 6, 7 e 8 evidenciam que o carregamento do sistema causa variação na amplitude das correntes de curto circuito em sistemas automatizados de distribuição de energia elétrica. Desta forma, torna-se necessário estudar formas de atenuar tais correntes faltosas.

\subsection{Métodos de atenuação das correntes de curto circuito}

Após a exposição dos dados até então obtidos, pode-se afirmar que os sistemas de distribuição de energia elétrica atuais (automatizados) necessitam de proteções com comportamento dinâmico, as

IX COEN - Congresso de Engenharias da UFSJ Interconexão. 


\section{Seja seu próprio

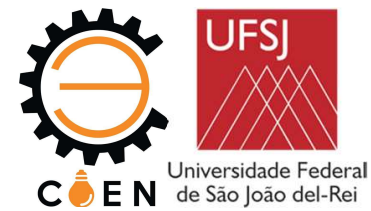

quais cobrem uma faixa de valores e não somente um valor específico, visto que as correntes elétricas assumem comportamento variável neste tipo de sistema. Logo, adiante serão expostos os três métodos propostos por este trabalho para atenuação destas correntes de curto circuito variáveis.

\section{1) Redespacho de potência reativa}

O método de redespacho de potência reativa foi implementado na rotina de simulação de correntes de curto circuito. Este método consiste na inserção de um banco de capacitores shunt na rede a fim de se encontrar a potência reativa necessária para injeção no momento da falta elétrica. Por meio de cálculos realizados, foi encontrado o valor de $75 \mathrm{kVAr}$ para cada módulo monofásico do banco de capacitores. Com a implementação destes, os níveis de correntes reduziram consideravelmente. Os valores obtidos estão expostos na Tabela 9.

Tabela 9. Correntes de curto circuito após a inserção do banco de capacitores

\begin{tabular}{ccc|ccc|ccc}
\hline \multicolumn{7}{c}{ Correntes de curto circuito entre as fases A e B [A] } \\
\hline \multicolumn{2}{c|}{ Carga leve (0,9 p.u.) } & \multicolumn{2}{c|}{ Carga moderada (1,7 p.u.) } & \multicolumn{3}{c}{ Carga máxima (2,4 p.u.) } \\
\hline Fase A & Fase B & Fase C & Fase A & Fase B & Fase C & Fase A & Fase B & Fase C \\
\hline & & & & & & & & \\
471,29 & 471,29 & 0 & 473,07 & 473,07 & 0 & 475,82 & 475,82 & 0 \\
\hline
\end{tabular}

\section{2) Inserção de reatores em série}

Este método consiste na especificação de reatores em série com o circuito, a fim de limitar as correntes de curto circuito aumentando a impedância vista pela falta elétrica. Para simular a inserção deste dispositivo na rede de distribuição foi necessário estabelecer um valor de indutância nominal.

Deste modo, para fins de simulação, foi escolhido um valor comercial já aplicado em sistemas de distribuição, como forma apenas de verificar a influência do mesmo no sistema. Os reatores monofásicos têm como valor de indutância nominal $L=0,58 \mathrm{mH}$. Logo, a reatância inserida corresponde ao valor exposto na Equação 1.

$$
X_{L}=2 \times \pi \times 60 \times(0,58 \times 10-3)=0,22 \Omega
$$

Esta reatância obtida foi somada à impedância de Thèvenin para a obtenção das novas correntes de curto circuito. Os novos valores de corrente são expostos pela Tabela 10.

Tabela 10. Correntes de curto circuito após a inserção de reatores.

\begin{tabular}{ccc|ccc|ccc}
\hline \multicolumn{7}{c}{ Correntes de curto circuito entre as fases A e B [A] } \\
\hline \multicolumn{2}{c|}{ Carga leve (0,9 p.u.) } & \multicolumn{3}{c|}{ Carga moderada (1,7 p.u.) } & \multicolumn{3}{c}{ Carga máxima (2,4 p.u.) } \\
\hline Fase A & Fase B & Fase C & Fase A & Fase B & Fase C & Fase A & Fase B & Fase C \\
\hline & & & & & & & & \\
537,02 & 537,02 & 0 & 541,49 & 541,49 & 0 & 547,01 & 547,01 & 0 \\
\hline
\end{tabular}

3) Comutador Eletrônico de Taps

IX COEN - Congresso de Engenharias da UFSJ Interconexão. 

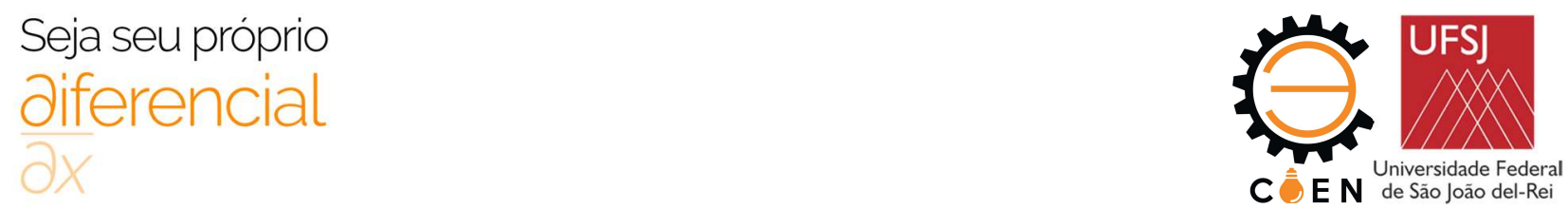

Por sua vez, este método tem como intuito o chaveamento instantâneo dos taps do regulador de tensão, como forma de aumentar a impedância vista pela corrente no momento da falta elétrica. $\mathrm{O}$ método é realizado por meio de um comutador eletrônico de taps (CET), o que torna possível esta aplicação na prática, pois os comutadores eletromecânicos não conseguem realizar operações instantâneas. Os resultados obtidos em função deste método estão expostos na Tabela 11.

Tabela 11. Correntes de curto circuito após a inserção do comutador eletrônico de taps.

\begin{tabular}{ccc|ccc|ccc}
\hline \multicolumn{7}{c}{ Correntes de curto circuito entre as fases A e B [A] } \\
\hline \multicolumn{2}{c|}{ Carga leve (0,9 p.u.) } & \multicolumn{2}{c|}{ Carga moderada (1,7 p.u.) } & \multicolumn{3}{c}{ Carga máxima (2,4 p.u.) } \\
\hline Fase A & Fase B & Fase C & Fase A & Fase B & Fase C & Fase A & Fase B & Fase C \\
\hline & & & & & & & & \\
4
\end{tabular}

4) Considerações sobre os métodos

Os métodos 1 e 3 realizaram a atenuação da magnitude das correntes de forma mais eficaz.

Independentemente do valor de carga, o comportamento do método 1 permaneceu estável na casa dos $17 \%$ de atenuação, com uma variação de $\pm 0,5 \%$. Como se trata de um banco de capacitores, é possível realizar um acréscimo no valor de cada módulo monofásico, aumentando assim, a potência reativa despachada no momento da falta, conseguindo resultados ainda mais expressivos quanto à redução da amplitude das correntes. Apesar dos bons resultados, o método apresenta um alto valor de investimento, visto que bancos de capacitores apresentam um preço elevado em relação aos dispositivos convencionais, que pode representar um entrave na aplicação do sistema do ponto de vista econômico.

O método 2 se mostrou pouco efetivo, já que a sua redução máxima foi de 5,38\%. Assim como o banco de capacitores, é possível aumentar o valor da sua indutância para obter uma melhoria de performance, ou até mesmo associações com mais de um reator por fase. No entanto, este método visa apenas a redução da corrente de curto circuito para o valor de capacidade de interrupção dos disjuntores de ajuste fixo. Sendo assim, sua proposta é de apenas realizar uma baixa redução na corrente, e o aumento do valor de indutância para reduções elevadas pode aumentar consideravelmente o custo de implantação desta metodologia, representando uma inviabilidade econômica.

Por fim, o método 3 apresenta algumas particularidades. Este método é o que apresentou a maior porcentagem de redução de corrente $(19,79 \%)$, quando submetido à um fator de carga leve. Porém, com o aumento do carregamento, o método foi perdendo sua capacidade de redução de corrente, uma vez que o tap já se situava em posições elevadas. Logo, sua aplicabilidade fica muito restrita à sistemas automatizados que não excedam seus pontos de carregamento médio.

Assim, a Tabela 12 sintetiza as vantagens e desvantagens de cada metodologia proposta. 

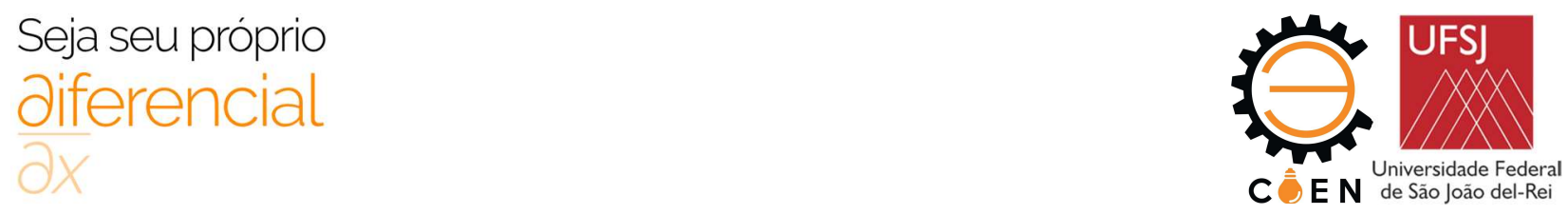

Tabela 12. Principais vantagens e desvantagens de cada método.

\begin{tabular}{|c|c|c|}
\hline \multicolumn{3}{|c|}{ Pontos relevantes de cada metodologia simulada } \\
\hline Método & Vantagens & Desvautagens \\
\hline I & $\begin{array}{l}\text { - Consegue atenuar as correntes de curto } \\
\text { circuito independentemente do fator } \\
\text { de carga } \\
\text { - Seu fator de redução apresenta pouca } \\
\text { variação }\end{array}$ & - Alto custo de implementação \\
\hline II & $\begin{array}{l}\text { - Fácil implementaçâo } \\
\text { - Método já sendo encontrado em } \\
\text { aplicaçôes práticas }\end{array}$ & $\begin{array}{l}\text { - Baixa atenuação dos niveis de corrente } \\
\text { - Para reduçöes consideráveis é necessário } \\
\text { realizar associaçōes de reatores, } \\
\text { aumentando o custo de implementação }\end{array}$ \\
\hline III & $\begin{array}{l}\text { - Apresenta altas reduçôes de corrente } \\
\text { para baixos fatores de carga } \\
\text { - Baixo custo de implementação }\end{array}$ & $\begin{array}{l}\text { - Perde eficácia à medida que o } \\
\text { carregamento aumenta } \\
\text { - Não pode serimplementado em sistemas } \\
\text { que atendem a um grande número de } \\
\text { cargas (urbanos, por exemplo) }\end{array}$ \\
\hline
\end{tabular}

\section{CONSIDERAÇÕES FINAIS}

Ressalta-se que todos os métodos propostos não visam a substituição de dispositivos de proteção que cessam as correntes de curto circuito, mas sim, uma ação conjunta com os mesmos, permitindo dinamizar um sistema constituído, originalmente, por ajustes fixos (disjuntores, relés, fusíveis, etc.). Uma vez que todos os métodos possuem um range de atuação, a falta elétrica poderá ocorrer em qualquer ponto dentro dos limites dos respectivos métodos, pois esta corrente de curto circuito será reduzida para o valor de atuação pré-estabelecido da proteção convencional e interrompida sem causar danos ao sistema.

Porém, o conceito de ajuste dinâmico não fica restrito apenas a estes métodos. Ainda se trata de um tema pouco abordado no Brasil, porém as smart grids podem tornar este conceito comum, visto que dispositivos eletromecânicos de proteção tendem a perder espaço para dispositivos eletroeletrônicos à medida que as smart grids forem se intensificando. Estes dispositivos eletroeletrônicos propiciam ajustes dinâmicos ao sistema de proteção, oferecendo critérios de seletividade e coordenação à proteção conforme seja a carga atendida e o tipo de falta elétrica ocorrida, mostrando que independentemente do método utilizado, o conceito de ajuste dinâmico é, cada vez mais, preponderante para sistemas de distribuição de energia elétrica.

\section{DIREITOS AUTORAIS}

Os autores são os únicos responsáveis pelo conteúdo das informações contidas neste artigo.

\section{REFERÊNCIAS}

ANEEL. Procedimentos de Distribuição de Energia Elétrica no Sistema Elétrico Nacional PRODIST: Módulo 8 - Qualidade de Energia Elétrica. Revisão, v. 10, 2018. Disponível em:<http://www.aneel.gov.br/documents/656827/14866914/M\%C3\%B3dulo_8Revis\%C3\%A3o_10/2f7cb8 62-e9d7-3295-729a-b619ac6baab9>. Acesso em: 22 de junho de 2019.

IX COEN - Congresso de Engenharias da UFSJ Interconexão. 

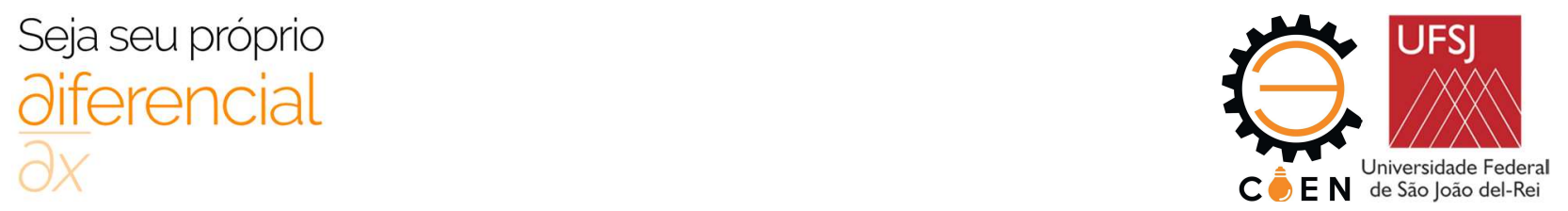

BURATTI, R. P. Reguladores de tensão em redes elétricas com alta penetração de cargas não lineares. Dissertação (Mestrado em Engenharia Elétrica), Faculdade de Engenharia do Campus de Ilha Solteira UNESP, 2016.

CONDEGA, S. Y. C. Metodologia para determinação da margem de estabilidade de tensão sob a perspectiva da expansão e da operação de sistemas de potência. Tese de Doutorado. Dissertação de Mestrado em Engenharia Elétrica, PPGEE/UFMG, 2013.

DE ALMEIDA, J. C. Comparação entre métodos e análise de correntes de curto circuito. Universidade Estadual Paulista, Faculdade de Engenharia de Guaratinguetá, 2012.

DE SOUZA, A. C. Z. et al. Using PV and QV curves with the meaning of static contingency screening and planning. Electric Power Systems Research, v. 81, n. 7, p. 1491-1498, 2011.

DOS REIS, C. S. M. M. Determinação de Índices de Estabilidade de Tensão. Mestrado (Dissertação), Faculdade de Engenharia da Universidade do Porto. Porto, 2005.

FURINI, M. A.; ARAUJO, P. B. de. Melhora da estabilidade dinâmica de sistemas elétricos de potência multimáquinas usando o dispositivo facts "thyristor-controlled series capacitorTCSC". Sba: Controle \& Automação Sociedade Brasileira de Automatica, v. 19, n. 2, p. 214-225, 2008.

GERS, J. M., HOLMES, E. J. Protection of Electricity Distribution Networks. Power and Energy Series 28, The Institution of Electrical Engineers - IEE, Reino Unido, 2005.

2002.

KERSTING, W. H. Distribution system modeling and analysis. CRC Press, 2nd ed., New York, USA,

KUNDUR, P. et al. Definition and classification of power system stability. IEEE transactions on Power Systems, v. 19, n. 2, p. 1387-1401, 2004.

LUIZ, C. M. Avaliação dos Impactos da Geração Distribuída para Proteção do Sistema Elétrico. Programa de Pós-Graduação em Engenharia Elétrica, Universidade Federal de Minas Gerais - UFMG, Escola de Engenharia, Belo Horizonte, 2012.

MEDEIROS, M. F.; PIMENTEL, M. C. Localização ótima de bancos trifásicos de reguladores de tensão em alimentadores radiais de distribuição. In: CONGRESSO BRASILEIRO DE AUTOMÁTICA-CBA. 2002.

MOURA, R. S. et al. Effects of QV curves in the dynamic behaviour of power systems. IET Generation, Transmission \& Distribution, v. 10, n. 12, p. 2861-2870, 2016.

OSORIO, E. A. A.; PAN, A. C. Dimensionamento de um sistema de aterramento em uma usina solar. VII Congresso Brasileiro de Energia Solar. Faculdade de Física da Pontifícia Universidade Católica - Rio Grande do Sul, Gramado, 2018.

\section{INFLUENCE OF VOLTAGE REGULATORS IN THE CALCULATION OF SHORT CIRCUIT CURRENTS IN ELECTRICITY DISTRIBUTION SYSTEMS}

Luís Henrique Rodrigues Diniz ${ }^{(1)}$ (luishenrique@eletroterraengenharia.com.br), Renan Souza Moura ${ }^{(2)}$ (renan.moura@ifmg.edu.br), Ulysses Rondina Duarte ${ }^{(3)}$ (ulysses.rondina@ifmg.edu.br), Carlos Renato Borges dos Santos ${ }^{(4)}$ (carlos.renato@ifmg.edu.br)

(1) (2) (3) (4) IFMG Campus Formiga - Departamento de Engenharia Elétrica - Rua Padre Alberico, 440, São Luiz, Formiga - MG.

ABSTRACT: This paper approaches the presence of variable short circuit currents in automated systems of electric power distribution. Usually, the adjustments of the protection system are fixed, therefore, they may

IX COEN - Congresso de Engenharias da UFSJ Interconexão. 
not contemplate any variation of current during coordination. However, the insertion of a voltage regulator changes the system impedance as a function of the tap position and this may compromise the correct operation of relays that aim to protect the electrical system. In this work, the influence of the variable short-circuit currents as a function of system loading and three computational methods, simulated in a Matlab environment, to control and attenuate these currents are analyzed: i) reactive power redispatch, ii) series reactor insertion and iii) electronic tap-change. In all simulations, the operacional limits of the analyzed system are considered. The results demonstrated that all of methods are effective in reducing variable shortcircuit currents: method i) around $17 \%$, method ii) around 5\% and method iii) around $19 \%$. Advantages and disadvantages of each method are discussed in this article. Although the presented computacional methods are not yet possible to be used in large-scale experimental systems, it is expected that this work will focus more attention on the dynamic adjustment of the protection system, especially with the intensification of investments in smart grids.

KEYWORDS: Variable short circuit currents. PV curve. Short circuit current control methods. 